\section{(6) OPEN ACCESS}

\title{
Second generation HIV surveillance in Pakistan: policy challenges and opportunities
}

\author{
Michelle Munro, ${ }^{1}$ Merydth Holte-McKenzie, ${ }^{1}$ Sadia Ahmed, ${ }^{2}$ Chris P Archibald, ${ }^{3}$ \\ James F Blanchard, ${ }^{4}$ Laura $\mathrm{H}$ Thompson ${ }^{4}$
}

${ }^{1}$ Agriteam Canada Consulting Ltd., Gatineau, Quebec, Canada

${ }^{2}$ Canadian High Commission, Islamabad, Pakistan

${ }^{3}$ Centre for Communicable Diseases and Infection Control, Public Health Agency of Canada, Ottawa, Ontario, Canada

${ }^{4}$ Centre for Global Public Health, Department of Community Health Sciences, University of Manitoba, Winnipeg, Manitoba, Canada

\section{Correspondence to} Dr James F Blanchard, Centre for Global Public Health, Department of Community Health Sciences, University of Manitoba, R070-771

McDermot Avenue, Winnipeg, MB R3E 0T6;

james_blanchard@umanitoba. ca

Accepted 16 September 2012 Published Online First

8 December 2012

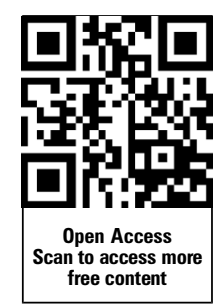

To cite: Munro M, HolteMcKenzie M, Ahmed S, et al. Sex Transm Infect 2013;89:ii48-ii52.

\begin{abstract}
From 2004 to 2011, the Canada-Pakistan HIV/AIDS Surveillance Project (HASP) worked with government and non-government partners in Pakistan to design and implement an HIV second generation surveillance (SGS) system. Insights into the development of scalable cost effective surveillance methodologies, implementation, use of data for HIV prevention and human rights were gained over the course of HASP. An ideal SGS system would be affordable, able to be implemented independently by local partners and produce data that could be readily applied in policy and programmes. Flexibility in design and implementation is important to ensure that any SGS system is responsive to information needs, political changes and changes in key population dynamics and HIV epidemics. HASP's mapping methodology is innovative and widely accepted as best practice, but sustainability of the SGS system it developed is a challenge.
\end{abstract}

\section{INTRODUCTION}

Between 2004 and 2011, the Canada-Pakistan HIV/AIDS Surveillance Project (HASP) conducted four rounds of integrated behavioural and biological surveillance (IBBS) in Pakistan. A 7 year development project funded by the Canadian International Development Agency, HASP aimed to support the Government of Pakistan to establish a national HIV second generation surveillance (SGS) system. It provided Pakistan's National and Provincial AIDS Control Programmes, as well as local health research organisations and nongovernmental organisations (NGOs), with the requisite resources to conduct SGS, complemented by technical assistance from the Public Health Agency of Canada and the Universities of Manitoba and Montréal.

HIV surveillance is an essential part of monitoring and evaluation policy frameworks. Such frameworks ensure that methods provide the necessary information at the right frequency, and are ethical, acceptable, scalable and cost effective. ${ }^{1}$ First generation HIV surveillance monitored prevalence of infection and disease reports but, largely because of HIV's unique epidemiology, which can lead to multiple epidemics, was not suitable for all epidemic types and tended to exclude information such as risk behaviours. ${ }^{2}$ Thus the SGS approach, which tailors surveillance methods to different epidemic typologies, was developed. WHO and UNAIDS define SGS as the, '...regular, systematic collection, analysis and interpretation of information for use in tracking and describing changes in the HIV/ AIDS epidemic over time'. ${ }^{3}$ They outline three epidemic types-generalised, concentrated, and low level -and surveillance approaches to each. WHO/ UNAIDS define a concentrated epidemic as one in which prevalence is less than $1 \%$ in the general population but $5 \%$ or more among sub-populations at higher risk.

Pakistan's HIV epidemic is characterised by high HIV prevalence among those who inject drugs ${ }^{4}$ as well as significant rates among hijra (transgender), male and female sex workers, but few infections in the wider population. In concentrated epidemics such as Pakistan's, behavioural information is particularly important as it can help to predict the extent to which sub-populations at the highest risk engage in risk behaviours with individuals outside these sub-populations, thus potentially leading to epidemic transition.

HASP generated rich experiences about how to design, conduct and translate knowledge from SGS in a concentrated epidemic. They may be applicable to a number of HIV surveillance and wider public health policy arenas. Four such arenas will be discussed in this paper: developing a scalable methodology; policy related to operationalising HASP's model; the ability of the SGS system to impact HIV prevention, treatment and care and support policies and programmes; and SGS and human rights.

\section{DEVELOPING METHODOLOGIES FOR LARGE-SCALE HIV SURVEILLANCE IN PAKISTAN}

The HASP SGS system was designed to monitor the HIV epidemics in Pakistan and provide data for policy makers to use when reporting on national HIV targets, while also providing data useful for the development and evaluation of HIV prevention policy and programmes. Specifically, the locations and sizes of populations of injection drug users and male, hijra and female sex workers were estimated, after which data about behavioural risks, vulnerabilities and HIV prevalence were collected among them. This was conducted in four separate rounds and in many cities across Pakistan, allowing for comparisons and the analysis of temporal trends (box 1).

In order to develop the most appropriate and effective SGS system for Pakistan, a number of decisions had to be made about inclusion criteria, sampling, survey instruments and funding. First, decisions about the inclusion of particular key populations and sentinel sites were made. Cities with evidence of an HIV epidemic were selected as sentinel sites ${ }^{5}$ and key populations with evidence or plausibility of HIV transmission (injection drug users and male, hijra and female sex workers) were 
Box 1 Decisions made for the development of the methodology of the HIVIAIDS Surveillance Project

- Populations and sentinel sites

- Mapping versus extrapolation from surveys

- Sample sizes to reflect national, provincial or smaller jurisdiction HIV epidemic trends

- Amount of detail in behavioural survey

- Frequency of surveillance rounds

- Standardised instruments versus combining local surveys

- Dried blood spot versus whole blood testing

- Financing surveillance through donors or national government sources.

included. Agreement was reached after considerable and ongoing debate. Some stakeholders continue to suggest that Pakistan's SGS system should include men who have sex with men (MSM); HASP epidemiologists contend that the trends among male and transgender sex workers reflect and are an early warning for the trend among MSM. There was frequent pressure to include additional cities, and HASP did accommodate certain well-justified requests, such as to provide baseline data for new HIV prevention programmes. Although the sentinel site model was retained, flexibility was important to maximise the impact of the SGS.

The mapping methodology the HASP team developed has been recognised as best practice and incorporated by WHO advice for the region. ${ }^{6}$ The timing was opportune, as it coincided with similar efforts in other countries with concentrated epidemics, thus allowing HASP to contribute its mapping methodology to countries with similar epidemics and contexts.

HASP's mapping methodology first identifies locations where activities that increase the risk of HIV transmission occur and then estimates the number of key population members engaging in such activities. Initial mapping collects secondary data from key informants, which is then validated through interviews with and observation of key population members. As these populations are often marginalised and/or mobile, surveillance teams need to build trust and work closely with the pertinent populations while maintaining rigour and working rapidly to avoid double-counting. The methodology efficiently estimates the size of key populations without the need for large surveys and eliminated the need to rely on extrapolation from few studies of varied quality, although it is more resource intensive and costly compared with statistical extrapolation. ${ }^{7}$ Training field teams to conduct the mapping data collection is time-consuming, but given the concentrated nature of Pakistan's epidemic, the focus of mapping is narrow and therefore feasible.

There was much debate over whether sampling should be designed to meet the national- or the provincial-level needs. Data from smaller and simpler samples could have been obtained quickly at low cost to provide national level estimates, while avoiding security risks to which Pakistan is prone. However, health is administered at the provincial level and therefore stratified samples from multiple locations within the provinces have provided useful information for health programme planning. A flexible approach enabled the project to meet both national and provincial needs using a range of sampling strategies.

The behavioural surveys provided rich data sets by including questions capturing demographics, education, migration, income, housing, detailed risk behaviours, and HIV knowledge and risk reduction behaviours including indicators identified by the United Nations General Assembly Special Session (UNGASS) on HIV. The scope was intended to enable making predictions about the trajectory of the HIV epidemics and inform policy and programme planning. The development of the questionnaire was a participatory process with input from experts in HIV epidemiology and prevention, policy makers, NGO service providers, and members of key population communities; it was meant to meet the needs of them all. This increased the complexity of questionnaire development, and limiting its length was difficult. Indeed it grew significantly over the duration of HASP.

Behavioural and biological surveillance was conducted on a close-to-annual basis in order to capture shifts in the epidemics that occur quickly. The brief interval between surveillance rounds did not always leave sufficient time for provinces and NGOs to plan and implement HIV prevention programmes, let alone time for these programmes to have effects. Data generated through HASP have the potential to be used to improve HIV prevention programming, but adequate time must be allocated for planning and implementation. While the richness of HASP's data combined with its comparability between provinces was one of its strengths, inadequate capacity of key provincial and NGO staff posed a challenge for programme development and implementation.

The decision was made to develop a standard questionnaire for each key population and specify the data entry and analysis software to use. This decision was made because experiences in early rounds of HASP SGS demonstrated that autonomy over the process of data entry and analysis did not ensure data quality or timely analysis. Data entry took place at the provincial level, while data cleaning and analysis was carried out centrally by HASP to ensure consistency and quality. It can be a challenge to require the use of standardised methodologies while also promoting local collaboration and uptake of surveillance results. Even when there is consensus, policy frameworks and guidelines may need to be adapted to local needs and regulated. One option is to develop minimum standards for mapping and IBBS to allow for shorter, less costly but adequately rigorous studies to be conducted locally, which may be compared to the results of larger, periodic rounds of surveillance. An alternative option could be an even leaner national SGS methodology complemented by smaller local surveys focused on specific local data needs.

To effectively reach marginalised populations in Pakistan, they must be sought out in their communities, which produced a logistical challenge for HIV testing. A relatively new application of an older technology that increased the feasibility of fieldbased surveillance was the use of dried blood spot (DBS) technology to collect a capillary blood sample for HIV testing. ${ }^{8}$ While it is now the specimen collection method of choice by WHO/UNAIDS for a variety of surveillance purposes, this use of DBS was relatively new when the project began. It allowed for non-medical personnel to conduct biological surveillance activities in non-clinical sites, as well as collection of sufficient samples for quality assurance and a specimen repository for future analysis and research. However, as no validated laboratory test exists to identify active syphilis infection using DBS specimens, syphilis testing was excluded. There was significant discussion regarding this decision, but ultimately agreement that the challenges of whole blood data collection (to permit testing for active syphilis) outweighed the potential benefits of collecting anonymous syphilis data among the key populations HASP was surveying. 
Mid-project political events led to uncertainty regarding the government department responsible for storage, integrity and appropriate sharing of SGS data. The major policy lesson is for comprehensive data ownership and intellectual property plans be devised and agreed upon at the beginning of any similar initiative, along with mechanisms for updating them should organisational changes occur.

Countries are encouraged to develop strategies for resource mobilisation, incorporating internal and donor funding sources. ${ }^{1}$ In Pakistan there is heavy reliance on donor funding, and indeed this is how the SGS was funded. Financing a surveillance system through international donors is risky, as funding may be unpredictable, and moreover, donors may request changes in the surveillance methodologies and inclusion criteria. Gaps in funding and different methodologies may limit temporal trend analysis and geographical comparisons. Moreover, with global cuts in both development assistance and HIV prevention, HIV surveillance funding may become more difficult to secure.

Best practice in HIV SGS methodologies change over time as new methods are developed and HIV epidemics shift. To keep surveillance methods up to date, they need to be revisited regularly. HASP accomplished this by developing a system and guidelines for mapping and IBBS that were flexible and that have contributed to policy frameworks and guidelines on HIV monitoring and evaluation in Pakistan and regionally.

\section{OPERATIONALISING SGS: DEVELOPING SURVEILLANCE CAPACITY}

In addition to designing and implementing national SGS, HASP was intended to build government and non-governmental partners' capacity in the management of SGS systems and use of surveillance data to inform policy and programmes (box 2). Therefore, the principles guiding the operation of the SGS included building capacity in addition to feasibility and scalability. In addition, the funder required that partner NGOs and research organisations, selected to conduct the surveillance field work, be chosen through an annual competitive process. Operationalising SGS at a national scale through competitive procurement, while building sustainable capacity, proved to be a challenge that provided many lessons.

The project's capacity-building strategy with government partners involved HASP staff working with national and provincial counterpart staff. Government counterparts participated in SGS contracting, training of contractors and monitoring of field work, ensuring compliance with the protocol. HASP also proactively engaged its government partners in the interpretation and application of data. The National and four Provincial AIDS Control Programmes did demonstrate increased, albeit varying, engagement in the process of managing the SGS system. However, uncertainty in Pakistan's HIV governance structure,

Box 2 Options and approaches for operationalising second generation surveillance

- Build government capacity to manage second generation surveillance

- Develop approaches for capacity building on non-governmental organizations and research partners

- Contract data collection to non-governmental organisations and research institutes versus direct delivery. funding limitations and human resource changes meant that continuity among government counterparts was sub-optimal. The periodic nature of surveillance posed a challenge to maintaining engagement and capacity that had been built, and HASP staff may have been perceived as a resource available to manage the SGS system under government direction, rather than a resource to transfer knowledge and build capacity. While this was a challenge, it also presents an opportunity to re-consider the policies that guide project design, such as whether highly technical projects such as HASP should rethink capacity development approaches when resources, in particular human resources, are constrained. One option used by some donors is to allow funding to support salaries. ${ }^{9}$ Building capacity in resource mobilisation to ensure predictable financing is another. In addition, if the likelihood of sustained technical expertise within a public sector organisation is low, alternative approaches to project design could include building links with agencies that have expertise while also developing public sector skills in managing relationships with technical organisations.

HASP combined formal training on the SGS methodology and ethics with accompaniment to build the surveillance capacity of NGOs and research organisations successful in the tenders to perform the field work of the SGS system. Accompaniment provides experiential learning opportunities along with mentoring and coaching techniques. It is recognised by development professionals as more likely to be sustainable than training alone. ${ }^{10}$

Competitive, transparent, merit-based tendering to select NGOs and research institution partners was meant to counter corruption, which is documented to be high within Pakistan's health sector. ${ }^{11}$ In a review of corruption in HIV programmes, Transparency International identified government procurement of HIV services as an area in which corruption is likely to occur. $^{12}{ }^{13}$ However, tendering should be accompanied with other recognised anti-corruption measures such as strategies to increase civil society governance capacity, enhance political accountability, restrain power, and reform the public sector. Although these actions were beyond HASP's mandate, when issues related to transparency in procurement were encountered they were addressed as best as possible. Some organisations which competed through this process may have been more motivated by the funding opportunity than the vision to implement a high quality SGS. Therefore while tendering does comply with development principles and policy with respect to prevention of corruption, ${ }^{12}$ simultaneoususe as a capacity development strategy may not have been the optimal approach. Research on corruption prevention policy and measures is now widely recognised as being needed for HIV programmes; monitoring and evaluation, including SGS, is no exception. A valid question is whether the time expended on tendering and contract management affected the project's ability to operationalise SGS and build capacity in it. In one round, HASP compared the efficiency and rigour of SGS when HASP directly engaged field research staff, as compared to when HASP contracted local organisations. Using the direct approach, there were fewer difficulties in assuring rigour and protocol adherence. The training model, with some adaptations, was effective for both. Theoretically, directly hiring and supervising field researchers might have allowed for more innovation in methodologies. However, training, monitoring, and overseeing SGS overwhelmed HASP staff, and therefore would require a larger staff if used at scale.

Tendering issues decreased the attention HASP staff were able to provide to building quality SGS capacity, highlighting the 
need to find optimal strategies for building broad-based SGS capacity while also discouraging corruption. Working with one or two research organisations may have overcome some of these challenges and would have reduced the transaction costs of tendering. Overall, finding the right capacity development strategy while scaling up SGS through competitive tendering was a challenge.

\section{USING SGS DATA TO INFORM HIV PREVENTION POLICY AND PRIORITIES}

One of the goals of HASP was to facilitate the uptake of surveillance data for HIV prevention policy development and programme design. HASP SGS data informed two revisions of HIV policy in the National Strategic Framework and several funding applications, evidence that HASP influenced and created opportunities for better HIV policy and programmes. However, the data have not been used to their full potential. Although often underfunded, the important role of civil society in delivering HIV prevention services, in particular for key populations, has been well-established. ${ }^{14}{ }^{15}$ HASP planned to work with NGOs that provide HIV services to key populations at risk of HIV to help these organisations to use SGS data to inform their programmes. However, but cuts in NGO funding precluded this initiative, a missed opportunity which is regretted. Also, the skill set required to build SGS capacity is different from that needed to develop HIV programming capacity within NGOs. In the future, it may be useful to have separate mechanisms and teams working with government and NGOs, with opportunities to bring these groups together for cross-learning.

HIV programmes, including monitoring, evaluation and SGS, are increasingly being encouraged to integrate with wider health systems ${ }^{15}$ to enhance the affordability of HIV responses and enable adequate funding for them. ${ }^{16}$ Integration could present a potential opportunity to share resources and expertise in the longer term, and globally health systems are shifting towards integration. However, there is a risk that targeted HIV prevention could be subsumed within programmes for the wider population. Given the likelihood that HIV prevention will be underfunded in the foreseeable future and the crucial importance of targeted prevention, the best option may be to engage productively in integration planning so the challenges to HIV programmes and SGS are considered and addressed.

\section{HUMAN RIGHTS AND HIV SURVEILLANCE}

Pakistan is a signatory to the Universal Declaration of Human Rights, the Convention on the Rights of the Child and the Convention to Eliminate All Forms of Discrimination Against Women. However, Pakistan's progress in implementing these principles has been limited. Colonial legislation that continues to exist as well as Islamic law sanction criminal penalties for non-marital sex, sodomy, and commercial sex work. ${ }^{17}$ According to the most recent Human Development Index women's development is only $70 \%$ of men's. ${ }^{18}$ The key populations among which HASP surveillance was conducted have few legal or societal protections for their health, safety, confidentiality and other rights and this was considered in the design of the SGS methodologies.

Consistent with international standards, HASP used unlinked anonymous testing (UAT). ${ }^{19}$ Stakeholders in Pakistan encouraged HASP to link results in order to identify positive individuals and theoretically refer them to services. However, HASP technical advisors provided several rationales for using UAT instead of linked testing, including the need to avoid recruitment or selection bias skewing the results, and to prevent the purpose of surveillance being confused with service provision (indeed HASP surveyed only a small proportion of the key populations). Moreover, HASP managers felt that in Pakistan's highly politicised environment, combined with an often aggressive legal system, keeping participant names and HIV status private would have been a challenge.

Surveillance participants received information about where to obtain HIV testing and counselling to protect their rights to information and health. However, in some of these cities services were not locally available and participants needed to be referred to services some distance away.

To protect participants' rights to safety and confidentiality, sensitisation sessions were held early on with local police, municipal authorities, religious leaders, NGO partners, and community gatekeepers. Organisations to which participants could be referred for HIV counselling and testing were identified. Surveillance methodologies and security procedures were designed to ensure the safety and confidentiality of participants, through consultation with key population members. Meaningful engagement means sharing decision making, difficult in a research context, and consultation was not always able to provide key populations with true influence on methodologies. $^{20}$

Generally, over the project's life there was increased recognition of the ethical challenges in HIV surveillance among key populations at higher risk of HIV, again underlining the need for frequent reassessment and renewal of rights and ethical considerations and measures. Indeed, in the final year of HASP, guidelines to help SGS staff and partners understand and address gender inequality among sexual minorities were developed, informed by the patterns of vulnerability among key populations in Pakistan.

\section{CONCLUSIONS}

Through HASP, many lessons were learned about the design and implementation of SGS. It is important that surveillance be designed with potential for scale up and long-term sustainability in mind. An ideal system would be affordable, able to be implemented independently by local partners, and produce data that could be readily applied in policy and programmes. The need for flexibility in design and implementation resonated throughout the experience of HASP and is important to ensure that any SGS system is responsive to information needs, political changes, and changes in the key population dynamics and HIV epidemics. HASP's mapping methodology is innovative and widely accepted as best practice; institutionalising the SGS system the project developed at all stages of the research and knowledge translation cycle has been more challenging. However, HASP has produced a wealth of knowledge about key populations and HIV in Pakistan, and there is great potential for it to be translated into effective HIV prevention policy and programmes. While no new data collection is planned, the Government of Pakistan continues to collaborate with the University of Manitoba to refine analyses and better use the existing data to improve programming.

Acknowledgements We thank the HASP surveillance team for their efforts in mapping, data collection, specimen collection, data entry, and knowledge translation. We also thank the participants who took part in the surveys for their time and participation.

Contributors All authors contributed to the development and/or preparation of this manuscript. MM was primarily responsible for the development of this article. LHT critically reviewed and revised the drafts. MH-M, SA, CPA and JFB were involved in the conception of the article and critically reviewed the drafts.

Competing interests None. 
Ethics approval HOPE International in Pakistan and the Public Health Agency of Canada.

Provenance and peer review Commissioned; externally peer reviewed.

Open Access This is an Open Access article distributed in accordance with the Creative Commons Attribution Non Commercial (CC BY-NC 3.0) license, which permits others to distribute, remix, adapt, build upon this work non-commercially, and license their derivative works on different terms, provided the original work is properly cited and the use is non-commercial. See: http://creativecommons.org/ licenses/by-nc/3.0/

\section{REFERENCES}

1 The Global Fund to Fight AIDS, Tuberculosis and Malaria. Monitoring and Evaluation Toolkit: HIV, Tuberculosis and Malaria and Health Systems Strengthening. 3rd edn. 2009. http://www.rbm.who.int/toolbox/tool_MEtoolkit.html (accessed 4 Sep 2012).

2 World Health Organization and Joint United Nations Programme on HIV/AIDS. Guidelines for Second Generation HIV Surveillance for HIV: The Next Decade, 2000. http://www.searo.who.int/LinkFiles/Facts_and_Figures_01_2ndgen_Eng.PDF (accessed 4 Sep 2012).

3 WHO. Second generation surveillance for HIV. http://www.who.int/hiv/topics/ surveillance/2ndgen/en/, (accessed 30 Aug 2011).

4 NACP. 2010. UNGASS Pakistan Report: Progress Report on the Declaration of Commitment on HIVIAIDS for the United Nations General Assembly Special Session on HIV/AIDS, 2010.

5 Losos JZ. Routine and sentinel surveillance methods. Eastern Mediterranean Health Journal 1996:2:46-50.

6 WHO. HIV surveillance in the Middle East and North Africa: a handbook for surveillance planners and implementers, 2010.

7 Saidel T, Loo V, Salyuk T, et al. Applying current methods in size estimation for high risk groups in the context of concentrated epidemics: lessons learned. JHASE 2010;1:2.
8 World Health Organization and Joint United Nations Programme on HIVIAIDS. Guidelines for Using HIV Testing Technologies in Surveillance, 2001. http://data. unaids.org/publications/IRC-pub02/jc602-hivsurvguidel_en.pdf (accessed 4 Sep 2012).

9 Global Fund to Fight AIDS, Tuberculosis and Malaria. 2011. Guidelines for proposals: round 11

10 Eade D. Capacity building: an approach to people-centered development. UK and Ireland: Oxfam, 2007.

11 Pappas G, Ghaffar A, Masud T, et al. Governance and Health Sector Development: a case study of Pakistan. Internet I World Health Societal Politics 2009;7:1.

12 Transparency International. 2006. Corruption and HIV/AIDS. Working Paper 06/ 2006.

13 Vian T. Review of corruption in the health sector: theory, methods and interventions. Health Policy Plan 2008:23:83-93.

14 United Nations. Declaration of Commitment on HIV/AIDS. United Nations General Assembly Special Session on HIVIAIDS, 25-27 June 2001. New York, NY: United Nations, 2001.

15 Ooms G, Van Damme, Baber B, et al. The 'diagonal' approach to Global Fund financing: a cure for the broader malaise of health systems? Glob Health 2008;4:6.

16 Schwartländer B, Stover J, Hallett T, et al. Towards and improved investment approach for an effective response to HIVIAIDS. Lancet 2011;377:2031-41.

17 Government of Pakistan. Pakistan Penal Code (Act XLV of 1860). http://www. pakistani.org/pakistan/legislation/1860/actXLVof1860.html (accessed 4 Sep 2012).

18 United Nations Development Programme. Pakistan Country Profile: Human Development Indicators. http://hdrstats.undp.org/en/countries/profiles/PAK.html (accessed 4 Sep 2012).

19 Rennie S, Turner AN, Mupenda B, et al. Conducting Unlinked Anonymous HIV Surveillance in Developing Countries: Ethical, Epidemiological, and Public Health Concerns. PLoS Med 2009;6:e1000004.

20 International HIVIAIDS Alliance. 2006. All together now: community mobilization for HIVIAIDS. 